\title{
Evolutionary physical constants in Einstein's field equations and the redshift
}

\author{
Rajendra P. Gupta
}

Macronix Research Corporation, 9 Veery Lane, Ottawa, ON Canada K1J 8X4; guptarp@macronix.ca

\begin{abstract}
We have shown that the use of evolutionary gravitational constants $G$, the speed of light $c$, and cosmological constant $\Lambda$ in the Einstein's field equations leads to a very simple model that fits the supernovae Ia data with a single parameter as well as the standard $\Lambda$ CDM model with two parameters, and has the predictive capability superior to the latter. We have shown that at the current time $G$ and $c$ both increase as $\mathrm{d} G / \mathrm{dt}=5.4 G H_{0}$ and $\mathrm{d} c / \mathrm{dt}=1.8 c H_{0}$ with $H_{0}$ as the Hubble constant, and $\Lambda$ decreases as $\mathrm{d} \Lambda / \mathrm{dt}=-1.2 \Lambda H_{0}$. Negative finding on the variation of $G$ with time derived from the analysis of lunar laser ranging data in several studies has been attributed to the fact that these studies did not consider the variation of $c$ simultaneously with $G$.
\end{abstract}

Keywords: cosmology: theory, cosmological parameters - galaxies: distances and redshifts variable physical constants

\section{INTRODUCTION}

Varying physical constant theories have been in existence since time immemorial, especially since Dirac (1937, 1938) suggested such variation based on his large number hypothesis. The most comprehensive review of the subject was done by Uzan (2003) followed by his more recent review (Uzan 2011). Chiba (2011) has provided an update of the observational and experimental status of the constancy of physical constants. We therefore will not attempt to cover the subject's current status except to mention a few of direct relevance to this work. Magueijo $(2000,2003)$ is a strong proponent of the variable speed of light theories. Accordingly, the model developed here will be considered generally Lorentz invariant which is true for any theory based on varying constants. We will be using the null results of Hofmann and Muller's (2018) attempts to determine the variation of gravitational constant $G$ by analysing several decades of LLR data to establish that when the speed of light variation is included, one finds that $G$ and $c$ vary exactly in the proportion of 3 to 1 .

We will follow Maharaj and Naidoo's (1993) approach to include variable gravitational constant $G$ and cosmological constatnt $\Lambda$ in the Einstein's field equations, and generalize it to include the variable speed of light $c$, to obtain their solution for the Robert-Walker metric in Sec. 2. We will show that $\dot{\Lambda} / \Lambda=-1.2 H$ and $\dot{H} / H=-0.6 H$ where $H$ is the Hubble parameter. In Sec. 3 we will establish that $\dot{G} / G=5.4 H$ and $\dot{c} / c=1.8 \mathrm{H}$. We will derive the expression for distance modulus $\mu$ vs. redshift $z$ in Sec. 4 . Sec. 5 is devoted to state the methodology for fitting the supernovae Ia data (Scolnic et al. 2018) and apply the same to the new model and compare it with the fits obtained with the standard $\Lambda$ CDM model. In the final section, Sec. 6, we will discuss our findings and present conclusions.

The essence of this work is that the new model implicitly includes the cosmological constant (that is varying) and it can fit the supernovae Ia data with a single parameter almost as well as the $\Lambda \mathrm{CDM}$ model with two parameter, and it has the predictive capability superior to the latter. In addition, we not only establish that the physical constants vary, we provide exactly how much they vary.

\section{EVOLUTIONARY CONSTANTS MODEL}

We will develop our model in the general relativistic domain starting from the Robertson-Walker metric with the usual coordinates $x^{\mu}(c t, r, \theta, \phi)$ :

$d s^{2}=c^{2} d t^{2}-a(t)^{2}\left[\frac{d r^{2}}{1-k r^{2}}+r^{2}\left(d \theta^{2}+\sin ^{2} \theta d \phi^{2}\right)\right] .(1)$

Here $a(t)$ is the scale factor and $k$ determines the spatial geometry of the universe: $k=-1$ (closed), 0 (flat), +1 (open). The Einstein's field equations may be written in terms of the Einstein tensor $G^{\mu \nu}$, metric tensor $g^{\mu \nu}$, and energy-momentum tensor $T^{\mu \nu}$, as:

$G^{\mu \nu}+\Lambda g^{\mu \nu}=-\frac{8 \pi G}{c^{4}} T^{\mu \nu}$

When solved for the Robertson-Walker metric, we get the following non-trivial equations for the flat universe $(k=0)$ of interest to us here, with $p$ as the pressure and $\varepsilon$ as the energy density: 
$\frac{\ddot{a}}{a}+\frac{1}{2}\left(\frac{\dot{a}}{a}\right)^{2}=-\frac{4 \pi G}{c^{2}} p+\frac{1}{2} \Lambda$

$\frac{\dot{a}^{2}}{a^{2}}=\frac{8 \pi G}{3 c^{2}} \varepsilon+\frac{1}{3} \Lambda$

If we do not regard $G, c$ and $\Lambda$ to be constant and define $K \equiv G / c^{2}$, we may easily derive the continuity equation by taking time derivative of Eq. (4) and substituting in Eq. (3)

$\dot{\varepsilon}+\frac{3 \dot{a}}{a}(\varepsilon+p)+\frac{\dot{K}}{K} \varepsilon+\frac{\dot{\Lambda}}{8 \pi K}=0$

It reduces to the standard continuity equation when $K$ and $\Lambda$ are held constant. And since the Einstein's field equations require that the covariant derivative of the energy-momentum tensor $T^{\mu \nu}$ be zero, we can interpret Equation (5) as comprising of two continuity equations (Maharaj \& Naidoo 1993), viz

$\dot{\varepsilon}+\frac{3 \dot{a}}{a}(\varepsilon+p)=0$, and

$8 \pi \varepsilon \dot{K}+\dot{\Lambda}=0$

This separation simplifies the solution of the field equations (Eqs. 3 and 4). Eq. (6) yields the standard solution for the energy density $\varepsilon=\varepsilon_{0} a^{-3(1+w)}$. Here $w$ is the equation of state parameter defined as $p \equiv w \varepsilon$ with $w=0$ for matter, $1 / 3$ for radiation and -1 for $\Lambda$.

It has been explicitly discussed by Magueijo in several of his papers (e.g. Magueijo 2000) that this approach is not generally Lorentz invariant albeit relativistic. Moreover, one should ideally use Einstein-Hilbert action to obtain correct Einstein's equations with variable $G$ and $c$. Our approach therefore may be considered quasi-phenomenological.

Since the universe expansion is determined by $H(t) \equiv \dot{a} / a$, it is natural to assume the time dependence of any time dependent parameter to be proportional to $\dot{a} / a$ - the so called Machian scenario (Magueijo 2003). Let us therefore write

$\frac{\dot{K}}{K}=k\left(\frac{\dot{a}}{a}\right), \frac{\dot{\Lambda}}{\Lambda}=l\left(\frac{\dot{a}}{a}\right)$ and $\frac{\dot{H}}{H}=m\left(\frac{\dot{a}}{a}\right)$, i.e.

$K=K_{0} a^{k}, \Lambda=\Lambda_{0} a^{l}$ and $H=H_{0} a^{m}$.

Here $k$ (not the same as in Eq. (1)) $l$ and $m$ are the proportionality constants, and subscript zero indicates the parameter value at current epoch $\left(t=t_{0}\right)$. With this substitution in Eq. (4) we may write

$\frac{\dot{a}^{2}}{a^{2}}=H_{0}^{2} a^{2 m}=\frac{8 \pi}{3}\left(K_{0} a^{k}\right) \varepsilon_{0} a^{-3(1+w)}+\frac{1}{3} \Lambda_{0} a^{l}$.

Comparing the exponents of $a$ of all the terms, we may write $2 m=k-3-3 w=l$, and with $w=0$ for matter, we have $2 m=k-3=l$. Thus, if we know $k$, we know $l$ and $m$.

We can now have a closed analytical solution of Eq. (10) as follows ( since $a\left(t_{0}\right) \equiv 1$ ):

$a(t)=\frac{a(t)}{a\left(t_{0}\right)}=\left(\frac{t}{t_{0}}\right)^{\frac{2}{3+3 w-k}} ; \frac{\dot{a}}{a}=\frac{2}{3+3 w-k} t^{-1}$ $\frac{\ddot{a}}{\dot{a}}=\left(\frac{\dot{a}}{a}\right)\left(1-\frac{3+3 w-k}{2}\right) ;$ and $-q \equiv \frac{\ddot{a} a}{\dot{a}^{2}}=\frac{-1-3 w+k}{2}$.

Here $q$ is deceleration parameter that does not depend on time, i.e. $q_{0}=q$. As we know the radiation energy density is negligible at present, so we need be concerned with the matter only solutions, i.e. with $w=0$.

The deceleration parameter $q_{0}$ has been analytically determined on the premise that expansion of the universe and the tired light phenomena are jointly responsible for the observed redshift, especially in the limit of very low redshift (Gupta 2018). One could see it as if the tired light effect is superimposed on the Einstein de Sitter's matter only universe rather than the cosmological constant. By equating the expressions for the proper distance of the source of the redshift for the two, one gets $q_{0}=-0.4$. Then from Eq. (12) we get $k=1.8$, and also $l=-1.2$, and $m=-0.6$. We thus have from Eq. (8) $\dot{K} / K=1.8 H, \dot{\Lambda} / \Lambda=-1.2 H$ and $\dot{H} / H=-0.6 H$.

\section{VARYING $G$ AND $c$ FORMULATION}

Having determined the value of $k=1.8$, and since the Hubble parameter is defined as $H=\dot{a} / a$, we may write from Eqs. (8) and (9)

$K=K_{0} a^{1.8}$, and $\frac{\dot{K}}{K}=1.8 H$

We may also write explicitly

$\frac{\dot{K}}{K}=\frac{\dot{G}}{G}-\frac{2 \dot{C}}{c}=1.8 H$

Taking $H$ at the current time as $H_{0} \simeq 70 \mathrm{~km} \mathrm{~s}^{-1} \mathrm{Mpc}^{-1}(2.27$ $\times 10^{-18} \mathrm{~s}^{-1}$ ) we get $\frac{\dot{K}}{K}=4.09 \times 10^{-18} \mathrm{~s}^{-1}=1.29 \times$ $10^{-10} \mathrm{yr}^{-1}$.

The findings from the Lunar Laser Ranging (LLR) data analysis provides the limits on the variation of $\dot{G} / G(7.1 \pm 7.6$ $\times 10^{-14}$ ) (Hofmann \& Müller 2018), which is considered to be about three orders of magnitude lower than expected. However, the LLR data analysis is based on the assumption that the speed of light is constant and non-evolutionary. If this constraint is dropped then the finding would be very different.

As is well known (Merkowitz 2010), a time variation of $G$ should show up as an anomalous evolution of the orbital period $P$ of astronomical bodies expressed by Kepler's 3rd law:

$P^{2}=\frac{4 \pi^{2} r^{3}}{G M}$

where $r$ is semi-major axis of the orbit, $G$ is the gravitational constant and $M$ is the mass of the bodies involved in the orbital motion considered. If we take time derivative of Eq. (15), divide by $P^{2}$ and rearrange, we get

$\frac{\dot{G}}{G}=\frac{3 \dot{r}}{r}-\frac{2 \dot{P}}{P}-\frac{\dot{M}}{M}$

If we write $r=c t$ then $\frac{\dot{r}}{r}=\frac{1}{t}+\frac{\dot{c}}{c}$. We may now rewrite Eq. (16) as 
$\frac{\dot{G}}{G}-\frac{3 \dot{C}}{c}=\frac{3}{t}-\frac{2 \dot{P}}{P}-\frac{\dot{M}}{M}$.

Since LLR measures the time of flight of the laser photons, it is the right hand side of Eq. (17) that is determined from LLR data analysis (Hofmann \& Müller 2018) to be $7.1 \pm 7.6 \times 10^{-14}$ and not the right hand side of Eq. (16).

Then, taking the right hand side of Eq. (17) as 0 and combining it with Eq. (14), one can solve the two equations and get $\dot{G} / G=5.4 H_{0}=3.9 \times 10^{-10} \mathrm{yr}^{-1}$ and $\dot{c} / c=1.8 H_{0}=$ $1.3 \times 10^{-10} \mathrm{yr}^{-1}$. It should be emphasized that both $\dot{G} / G$ and $\dot{c} / c$ are positive and thus both of them are increasing with time rather than decreasing as is generally believed (Barnes \& Dicke 1961, van Flandern 1975). This may be considered the most significant observational finding of cosmological consequences just by studying the Earth-Moon system.

\section{REDSHIFT VS. DISTANCE MODULUS}

The distance $d$ of a light emitting source in a distant galaxy is determined from the measurement of its bolometric flux $f$ and comparing it with a known luminosity $L$. The luminosity distance $d_{L}$ is defined as

$$
d_{L}=\sqrt{\frac{L}{4 \pi f}}
$$

In a flat universe the measured flux could be related to the luminosity $L$ with an inverse square relation $f=L /\left(4 \pi d^{2}\right)$. However, this relation needs to be modified to take into account the flux losses due to the expansion of the universe through the scale factor $a$, the redshift $z$, and all other phenomena which can result in the loss of flux. Generally accepted flux loss phenomena are as follows (Ryden 2017):

a. Increase in the wavelength causes the flux loss proportional to $1 /(1+z)$, and

b. In an expanding universe, an increase in detection time between two consecutive photons emitted from a source leads to a reduction of flux proportional to $a$, i.e. proportional to $1 /(1+z)$.

Therefore, in an expanding universe the necessary flux correction required is proportional to $1 /(1+z)^{2}$. The measured bolometric flux $f_{B}$ and the luminosity distance $d_{L}$ may thus be written as:

$f_{B}=L /\left[4 \pi d^{2}(1+z)^{2}\right]$, and

$d_{L}=d(1+z)$.

How does $d$ compare with and without varying $c$ ? Let us first consider the case of non-expanding universe. The distance from the point of emission at time $t_{e}$ to the point of observation at time $t_{0}$ may be written as $d_{c}=\int_{t_{e}}^{t_{0}} c d t$. Therefore for constant $c=c_{0}$

$d_{c_{0}}=c_{0} t_{0}\left(1-\frac{t_{e}}{t_{0}}\right)$

When $c=c_{0} a^{1.8}$, and since $a=\left(\frac{t}{t_{0}}\right)^{\frac{2}{1.2}}$ from Eq. (11), we may write

$d_{c}=c_{0} \int_{t_{e}}^{t_{0}}\left(\frac{t}{t_{0}}\right)^{3} d t=\frac{c_{0}}{t_{0}^{3}} \int_{t_{e}}^{t_{0}} t^{3} d t=\frac{1}{4} c_{0} t_{0}\left(1-\frac{t_{e}^{4}}{t_{0}^{4}}\right)$
The ratio of the two distances may be considered the normalization factor $F$ when using the variable $c$ in calculating the proper distance of a source. Since $a \equiv 1 /(1+z)$, we may write for the source of redshift $z$ with emission time $t_{e}$

$\frac{t_{e}}{t_{0}}=a(z)^{0.6}=(1+z)^{-0.6}$, and

$F(z)=4\left[1-(1+z)^{-0.6}\right] /\left[1-(1+z)^{-2.4}\right]$

Now the proper distance of the source with variable $c$ may be defined as (Ryden 2017)

$$
\begin{gathered}
d_{P_{c}}=\int_{t_{e}}^{t_{0}}\left(\frac{c}{a}\right) d t=\int_{t_{e}}^{t_{0}}\left(\frac{c_{0} a^{1.8}}{a}\right) d t=c_{0} \int_{t_{e}}^{t_{0}} a^{0.8} d t \\
=c_{0} \int_{t_{e}}^{t_{0}}\left(\frac{t}{t_{0}}\right)^{\frac{4}{3}} d t=\frac{3}{7} c_{0} t_{0}\left[1-\left(\frac{t_{e}}{t_{0}}\right)^{\frac{7}{3}}\right] .
\end{gathered}
$$

From Eq. (11) $H_{0} \equiv \dot{a} / a=(2 / 1.2) t_{0}^{-1}$. Therefore,

$d_{P_{c}}=\frac{1}{1.4}\left(c_{0} / H_{0}\right)\left[1-(1+z)^{-1.4}\right]$.

Thus the expression for $d$ to be substituted in Eq. (20) to determine the luminosity distance of the source is $d=d_{P_{c}} F$.

Since the observed quantity is distance modulus $\mu$ rather than the luminosity distance $d_{L}$, we will use the relation

$$
\begin{aligned}
\mu=5 & \log \left(d_{L}\right)+25 \\
=5 \log \left(\frac{1}{1.4}\right. & R_{0}\left(1-(1+z)^{-1.4}\right)+5 \log (F(z)) \\
& +5 \log (1+z)+25 .
\end{aligned}
$$

Here $R_{0} \equiv c / H_{0}$ and all the distances are in Mpc.

We will compare the new model, hereafter referred as VcG $\Lambda$ (variable $c, G$ and $\Lambda$ ) model, with the standard $\Lambda C D M$ model which is the most accepted model for explaining cosmological phenomena, and thus may be considered the reference models for all the other models. Ignoring the contribution of radiation density at the current epoch, we may write the distance modulus $\mu$ for redshift $z$ in a flat universe for the $\Lambda \mathrm{CDM}$ model as follows (Peebles 1993):

$$
\begin{gathered}
\mu=5 \log \left[R_{0} \int_{0}^{z} d u / \sqrt{\Omega_{m, 0}(1+u)^{3}+1-\Omega_{m, 0}}\right] \\
+5 \log (1+z)+25
\end{gathered}
$$

Here $\Omega_{0, m}$ is the current matter density relative to critical density and $1-\Omega_{m, 0} \equiv \Omega_{\Lambda, 0}$ is the current dark energy density relative to critical density.

\section{SUPERNOVAE Ia z- $\mu$ DATA FIT}

We tried the VcG $\Lambda$ model developed here to see how well it fits the best supernovae Ia data as compared to the standard $\Lambda \mathrm{CDM}$ model. The data fit is shown in Fig. 1. The VcG $\Lambda$ model requires only one parameter to fit all the data $\left(H_{0}=68.90 \pm\right.$ $0.26 \mathrm{~km} \mathrm{~s}^{-1} \mathrm{Mpc}^{-1}$ ) whereas the $\Lambda \mathrm{CDM}$ model requires two parameters $\left(H_{0}=70.16 \pm 0.42 \mathrm{~km} s^{-1} \mathrm{Mpc}^{-1}\right.$ and $\Omega_{m, 0}=$ $0.2854 \pm 0.0245)$.

The data used in this work is the so called Pantheon Sample of 1048 supernovae Ia (Scolnic et al. 2018) in the range of $0.01<z<2.3$. To test the fitting and predictive capability of 
the two models, we divided the data in 6 subsets: a) $z<0.5$; b) $z<1.0$; c) $z<1.5$; d) $z>0.5$; and e) $z>1.0$; and f) $z>1.5$. In addition, we considered the fits for the whole data. The models were parameterized with subsets a), b) and c). The parameterized models were then tried to fit the data in the subsets that contained data with $\mathrm{z}$ values higher than in the parameterized subset. For example if the models were parameterized with data subset a) $z<0.5$, then the models were fitted with the data subsets d) $z>0.5$, e) $z>1.0$ and f) $z>1.5$.

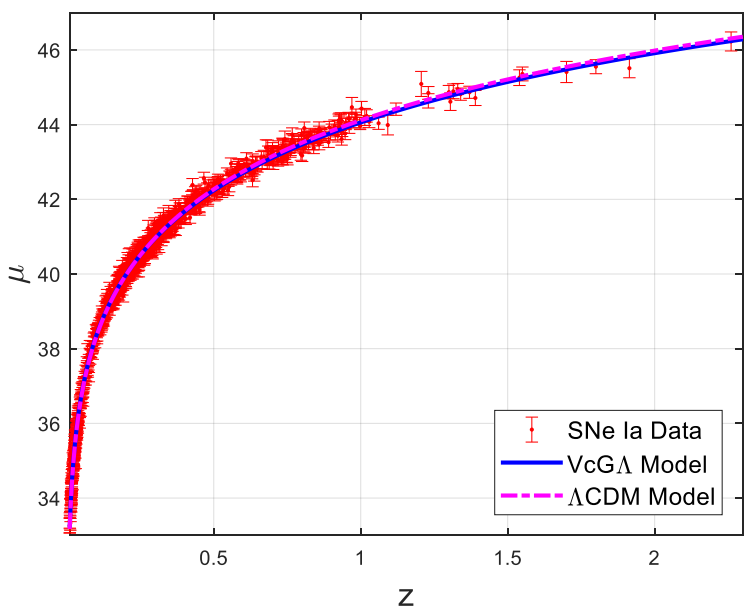

FIG. 1. Supernovae Ia redshift $z$ vs distance modulus $\mu$ data fit using the VcG $\Lambda$ model as compared to the fit using the $\Lambda C D M$ model.

The Matlab curve fitting tool was used to fit the data by minimizing $\chi^{2}$ and the latter was used for determining the corresponding $\chi^{2}$ probability $P$ (Press et al. 1992). Here $\chi^{2}$ is the weighted summed square of residual of $\mu$ :

$\chi^{2}=\sum_{i=1}^{N} w_{i}\left[\mu\left(z_{i} ; R_{0}, p_{1}, p_{2} \ldots\right)-\mu_{o b s, i}\right]^{2}$,

where $N$ is the number of data points, $w_{i}$ is the weight of the $i$ th data point $\mu_{o b s, i}$ determined from the measurement error $\sigma_{\mu_{O b s, i}}$ in the observed distance modulus $\mu_{o b s, i}$ using the relation $w_{i}=$ $1 / \sigma_{\mu_{O b s, i}}^{2}$, and $\mu\left(z_{i} ; R_{0}, p_{1}, p_{2} \ldots\right)$ is the model calculated distance modulus dependent on parameters $R_{0}$ and all other model dependent parameter $p_{1}, p_{2}$, etc. As an example, for the $\Lambda \mathrm{CDM}$ models considered here $p_{1} \equiv \Omega_{m, 0}$ and there is no other unknown parameter.

We then quantified the goodness-of-fit of a model by calculating the $\chi^{2}$ probability for a model whose $\chi^{2}$ has been determined by fitting the observed data with known measurement error as above. This probability $P$ for a $\chi^{2}$ distribution with $n$ degrees of freedom (DOF), the latter being the number of data points less the number of fitted parameters, is given by:

$P\left(\chi^{2}, n\right)=\left(\frac{1}{\Gamma\left(\frac{n}{2}\right)}\right) \int_{\frac{\chi^{2}}{2}}^{\infty} e^{-u} u^{\frac{n}{2}-1} d u$,

where $\Gamma$ is the well know gamma function that is generalization of the factorial function to complex and non-integer numbers. Lower the value of $\chi^{2}$ better is the fit, but the real test of the goodness-of-fit is the $\chi^{2}$ probability $P$; higher the value of $P$ for a model, better is the model's fit to the data. We used an online

TABLE I. Parameterizing and prediction table for the two models. This table shows how well a model is able to fit the data that is not used to determine the model parameters. The unit of $\mathrm{R}_{0}$ is $\mathrm{Mpc}$ and of $\mathrm{H}_{0}$ is $\mathrm{km} \mathrm{s}^{-1} \mathrm{Mpc}^{-1}$. $\mathrm{P} \%$ is the $\chi 2$ probability in percent that is used to assess the best model for each category; higher the $\chi 2$ probability $P$ better is the model fit to the data. $\mathrm{R}^{2}$ is the square of the correlation between the response values and the predicted response values. RMSE is the root mean square error. Highest $\mathrm{P} \%$ value in each category is shown in bold and the cell highlighted.

\begin{tabular}{|c|c|c|c|c|c|c|c|c|}
\hline Action / Item & $\Lambda \mathrm{CDM}$ & VcG $\Lambda$ & $\Lambda \mathrm{CDM}$ & VcG $\Lambda$ & $\Lambda \mathrm{CDM}$ & VcG $\Lambda$ & $\Lambda \mathrm{CDM}$ & VcG $\Lambda$ \\
\hline Parameterized & \multicolumn{2}{|c|}{ Model dataset $\mathrm{z}<0.5 ; 832$ points } & \multicolumn{2}{|c|}{ Model dataset $\mathrm{z}<1.0 ; 1025$ points } & \multicolumn{2}{|c|}{ Model dataset $\mathrm{z}<1.5 ; 1042$ points } & \multicolumn{2}{|c|}{ Model dataset all; 1048 points } \\
\hline $\mathrm{R}_{0}$ & $4259 \pm 34$ & $4337 \pm 18$ & $4269 \pm 27$ & $4351 \pm 17$ & $4271 \pm 26$ & $4352 \pm 17$ & $4273 \pm 26$ & $4351 \pm 16$ \\
\hline$\Omega_{\mathrm{m}, 0}$ & $0.2601 \pm 0.0457$ & NA & $0.2793 \pm 0.0261$ & NA & $0.2818 \pm 0.0249$ & NA & $0.2845 \pm 0.0245$ & NA \\
\hline $\mathrm{H}_{0}$ & $70.39 \pm 0.56$ & $69.13 \pm 0.29$ & $70.23 \pm 0.44$ & $68.90 \pm 0.27$ & $70.19 \pm 0.42$ & $68.89 \pm 0.27$ & $70.16 \pm 0.42$ & $68.90 \pm 0.25$ \\
\hline$\chi^{2}$ & 863.5 & 889.4 & 1018 & 1060 & 1033 & 1074 & 1036 & 1076 \\
\hline DOF & 830 & 831 & 1023 & 1024 & 1040 & 1041 & 1046 & 1047 \\
\hline $\mathrm{P} \%$ & 20.39 & 7.83 & 53.82 & 21.15 & 55.53 & 23.26 & 58.11 & 26.02 \\
\hline $\mathrm{R}^{2}$ & 0.9961 & 0.9960 & 0.9969 & 0.9968 & 0.9970 & 0.9969 & 0.9970 & 0.9969 \\
\hline RMSE & 1.020 & 1.035 & 0.9977 & 1.017 & 0.9965 & 1.016 & 0.9951 & 1.014 \\
\hline Model Fit & \multicolumn{2}{|c|}{ Dataset $\mathrm{z}>0.5 ; 216$ points } & \multicolumn{2}{|c|}{ Dataset $\mathrm{z}>0.5 ; 216$ points } & \multicolumn{2}{|c|}{ Dataset $\mathrm{z}>0.5 ; 216$ points } & \multicolumn{2}{|c|}{ Dataset $\mathrm{z}>0.5 ; 216$ points } \\
\hline$\chi^{2}$ & 176.9 & 190 & \multirow{5}{*}{\multicolumn{2}{|c|}{$\begin{array}{l}\text { NOT APPLICABLE SINCE } \\
\text { THIS DATASET INCLUDES } \\
\text { THE DATASET USED TO } \\
\text { PARAMETERIZE THE MODEL }\end{array}$}} & \multirow{5}{*}{\multicolumn{2}{|c|}{\begin{tabular}{|} 
NOT APPLICABLE SINCE \\
THIS DATASET INCLUDES \\
THE DATASET USED TO \\
PARAMETERIZE THE MODEL
\end{tabular}}} & \multirow{5}{*}{\multicolumn{2}{|c|}{$\begin{array}{l}\text { NOT APPLICABLE SINCE } \\
\text { THIS DATASET INCLUDES } \\
\text { THE DATASET USED TO } \\
\text { PARAMETERIZE THE MODEL }\end{array}$}} \\
\hline DOF & \multicolumn{2}{|c|}{216} & & & & & & \\
\hline $\mathrm{P} \%$ & 97.59 & 89.84 & & & & & & \\
\hline $\mathrm{R}^{2}$ & 0.9605 & 0.9575 & & & & & & \\
\hline RMSE & 0.905 & 0.938 & & & & & & \\
\hline Model Fit & \multicolumn{8}{|c|}{ Dataset $\mathrm{z}>1.0 ; 23$ points } \\
\hline$\chi^{2}$ & 19.54 & 17.01 & 17.59 & 16.75 & \multirow{5}{*}{\multicolumn{2}{|c|}{$\begin{array}{l}\text { NOT APPLICABLE SINCE } \\
\text { THIS DATASET INCLUDES } \\
\text { THE DATASET USED TO } \\
\text { PARAMETERIZE THE MODEL }\end{array}$}} & \multirow{5}{*}{\multicolumn{2}{|c|}{$\begin{array}{l}\text { NOT APPLICABLE SINCE } \\
\text { THIS DATASET INCLUDES } \\
\text { THE DATASET USED TO } \\
\text { PARAMETERIZE THE MODEL }\end{array}$}} \\
\hline DOF & \multicolumn{4}{|c|}{23} & & & & \\
\hline $\mathrm{P} \%$ & 66.94 & 80.43 & 77.93 & 82.13 & & & & \\
\hline $\mathrm{R}^{2}$ & 0.8741 & 0.8904 & 0.8867 & 0.8921 & & & & \\
\hline RMSE & 0.9216 & 0.86 & 0.8746 & 0.8533 & & & & \\
\hline Model Fit & \multicolumn{8}{|c|}{ Dataset $\mathrm{z}>1.5 ; 6$ points } \\
\hline$\chi^{2}$ & 4.090 & 1.946 & 3.167 & 1.983 & 3.076 & 1.986 & \multirow{5}{*}{\multicolumn{2}{|c|}{$\begin{array}{l}\text { NOT APPLICABLE SINCE } \\
\text { THIS DATASET INCLUDES } \\
\text { THE DATASET USED TO } \\
\text { PARAMETERIZE THE MODEL }\end{array}$}} \\
\hline DOF & \multicolumn{6}{|c|}{ 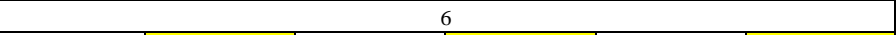 } & & \\
\hline $\mathrm{P} \%$ & 66.44 & 92.45 & 78.76 & 92.12 & 79.92 & 92.09 & & \\
\hline $\mathrm{R}^{2}$ & 0.5993 & 0.8093 & 0.6897 & 0.8057 & 0.6986 & 0.8054 & & \\
\hline RMSE & 0.8256 & 0.5696 & 0.7265 & 0.5749 & 0.716 & 0.5754 & & \\
\hline
\end{tabular}


calculator to determine $P$ from the input of $\chi^{2}$ and DOF (Walker 2018). Our primary findings are presented in Table I. The unit of the Hubble distance $R_{0}$ is $\mathrm{Mpc}$ and of the Hubble constant $H_{0}$ is $\mathrm{km} \mathrm{s}^{-1} \mathrm{Mpc}^{-1}$. The table is divided in four categories vertically and four categories horizontally. Vertical division is based on the parameterizing data subset indicated in the second row and discussed above. The parameters determined for each model are in the first horizontal category. The remaining horizontal categories show the goodness-of-fit parameters for higher redshift subsets than used for parameterizing the models. Thus this table shows the relative predictive capability of the two models. The model cells with the highest probability in each category are shown in bold and highlighted.

\section{DISCUSSION AND CONCLUSION}

As should be expected, the two parameter $\Lambda \mathrm{CDM}$ model is able to fit any data set better than the one parameter VcG $\Lambda$ model. What is unexpected is that when parameterized with a relatively low redshift data the $\mathrm{VcG} \Lambda$ model is able to fit the higher redshift data better than the $\Lambda$ CDM model. This shows that the second parameter in the latter, while trying to fit a limited dataset as best as possible, compromises the model fit for data not used for parameterizing. This means that the $\Lambda \mathrm{CDM}$ model does not have as good a predictive capability (i.e. the capability to fit the data that is not included for determining the model parameters) as the $\mathrm{VcG} \Lambda$ model despite having twice as many parameters as the VcG $\Lambda$ model. In addition the VcG $\Lambda$ model has the analytical expression for the distance $\mu$ unlike the $\Lambda \mathrm{CDM}$ model which must be evaluated numerically.

One would notice that while $R_{0}$ (and hence $H_{0}$ ) values are relatively stable with the parameterizing dataset containing higher and higher redshift values, varying no more than $0.35 \%$, while the variation in the $\Omega_{m, 0}$ is up to $9.4 \%$, i.e. 27 time higher. This confirms that $\Omega_{m, 0}$ parameter, and hence $\Lambda$ through $\Omega_{\Lambda, 0}$, is an artificially introduced parameter to fit the data rather than being fundamental to the $\Lambda$ CDM model. In contrast $\Lambda$ is an integral part of the VcG $\Lambda$ model. Since $K\left(\equiv G / c^{2}\right)$ and $\Lambda$ are related through Eq. (7), one could easily derive that the $\Lambda$ term contributes $60 \%$ for the $\mathrm{VcG} \Lambda$ model against $70 \%$ for the $\Lambda$ CDM model.

We have established that the supernovae 1a data is compatible with the variable constants proposition. This is contrary to the findings of Mould and Uddin (2014) who considered only the variation of $G$ in their work. We believe most of the negative findings on the variation of physical constants are possibly due to one considering the variation of a constant in isolation rather than holistically for all the constants involved. We have established that the physical constants not only vary but also how much they vary: $\dot{c} / c=1.8 H, \dot{G} / G=5.4 H$, $\dot{\Lambda} / \Lambda=-1.2 H$ and $\dot{H} / H=-0.6 H$. Also, from the null results on the variation of the fine structure constant (Gohar 2017, Rosenband et al. 2008) we can show that $\dot{\mathrm{h}} / \hbar=1.8 H$. We urge that they be used in union rather than in isolation.

Acknowledgements Author is thankful to Franz Hofmann for providing his latest research paper and references on Lunar Laser Ranging. He is grateful to Dan Scolnic for providing the SNe Ia Pantheon data used in this work.

\section{References}

Barnes, C., Dicke, R. H. 1961 Phys. Rev. 124, 925. Chiba, T. 2011, Prog. Theor. Phys. 126, \#6.

Dirac, P. A. M. 1937, Nature 139, 323.

Dirac, P. A. M. 1938, Proc. Roy. Soc. A 165, 199-208. Gohar, H. 2017, Universe 3, 26.

Gupta, R. P. 2018, Int'l J. Astron. Astrophys. 8, 219; Universe 4, 104.

Hofmann, F, Müller, J. 2018, Class. Quant. Grav. 35, 035015.

Magueijo, J. 2000, Phys. Rev. D 62, 103521.

Magueijo, J. 2003, Rept. Prog. Phys. 66, 2025.

Maharaj, S. D., Naidoo, R. 1993, Astrophys. Sp. Sci. 208, 261.

Merkowitz, S. M. 2010, Liv. Rev. Rel. 13, 7.

Mould, J., Uddin, S. A. 2014, PASA 31, e015.

Peebles, P. J. E. 1993, Principles of Physical Cosmology (Princeton University Press, N.J.).

Press, W. H., Teukolsky, S. A., Vetterling, W. T., Flannery, B. P. 1992, Numerical Recipes in C - The Art of Scientific Computing, $2^{\text {nd }}$ Ed. (Cambridge University Press, U. K.).

Rosenband, T., Hume, D. B., Schmidt, P. O., Chou, C. W., Brusch, A., Lorini, et al. 2008, Science 319, 1808.

Ryden, B. 2017 Introduction to Cosmology (Cambridge University Press, U.K.).

Scolnic, D. M., Jones, D. O., Rest, A., et al. 2018, ApJ. 859, 101.

Uzan, J-P. 2003, Rev. Mod. Phys.75, 403.

Uzan, J-P. 2011, Liv. Rev. Rel., 14, 2.

van Flandern, T. C. 1975, Mon. Not. Roy. Astron. Soc. $170,333$.

Walker, J. 2018 Chi-square calculator - https://www.fourmilab.ch/rpkp/experiments/analysis/chiCalc.html. 